\title{
Post-non-classical Ecology as a Direction in Modern Scientific- philosophical Research
}

\author{
Anatoly Stepanishchev, Dmitry Koshlakov ${ }^{1}$ \\ ${ }^{1}$ State Educational Institution of Higher Professional Education "Bryansk State Technical University", Department of Philosophy, \\ History \& Sociology, Bryansk, 241035, Russia
}

\begin{abstract}
In the context of the concept of post-non-classical knowledge developed by V.S. Styopin, a postnon-classical ecology as a certain direction of intelligent research and as a prospect in the development of modern scientific-philosophical knowledge is subjected to the analysis. A number of philosophical ideas belonging to the post-non-classical ecology are formulated, and in their sense they correspond to the concept of auto-poiesis. Some directions in the post-non-classical ecology are mentioned.
\end{abstract}

\section{Introduction}

Styopin's theory is the best known theory in Russian philosophy. It supposes, in particular, that sciences in general and separate sciences in particular pass in their development three stages : classical, non-classical and post-non-classical. The concept of post-non-classical knowledge allows comprehending, in a definite way, modern science and philosophy, their prospects and development.

In post-non-classical scientific knowledge V.S. Styopin discovers the integration of a subject, object and the instrument of cognition. Hence, the negotiation of subject and object called dualism was one of the dominants for gnoseology for a long time. In some wider interpretation the post-non-classical knowledge supposes the elimination of not only dualism, but other methodological dualisms characterizing scientific knowledge at the previous stages of development. Mr. Styopin, for instance, points out the negotiation of dualism of nature and culture in modern science [1].

From our point of view it is expedient to mention the formation of the community in scientific and philosophical rationalities as one more extremely significant characteristic of post-non-classical knowledge. Just in similar context one is required to consider the formation and development of general scientific knowledge (systems theory, synergy theory and so on), all parts of which belong to science and philosophy simultaneously. The most vivid example here could exemplify synergy of the methods, which have not only developed scientific and philosophical levels, but found their application in natural science, in engineering knowledge, and in social science and liberal arts. Ecological science is not an exclusion here. During some decades it has been attractng attention of scientific and world community, public organizations and politicians. It is naturally that in the context of post-non-classical trends growth a question of post-non-classical ecology development arises. Accordingly, a necessity to describe its most general features and prospects arises. The purpose of this paper consists in the outlining of basic features of the post-non-classical ecology as a certain arising phenomenon and as a certain prospect. It goes without saying, that the appearance of the post-nonclassical ecology, which we try to describe and concretize, to the considerable extent will be determined through the concept of post-non-classical and, in general, modern knowledge which we will use. In this thesis we relying upon the famous work by J. Delese and F.Guattari « What is Philosophy? » we proceed from the comprehension of philosophy as a concept creation [3]. Thereupon we mention that for us the post-non-classical knowledge is knowledge within the limits of which the integration of the rationalities of science and philosophy, as well as the negotiation of a number of methodological dualisms, affecting considerably science development at the stage of its classic and non-classic, occurs. Similar methodological dualisms (and in more general sense methodological oppositions) can be considered as one of significant factors of contradiction in scientific rationality. The matter concerns, for example, such dualisms as a binary opposition of natural and socialhumanitarian knowledge, contraposition of social science and liberal arts. The negotiation of these and other dualisms should contribute to the formation of unity in the science rationality.

\section{Results and discussion}

\footnotetext{
a Corresponding author: filosofiabryansk@mail.ru
} 
We agreed that one of the features in post-non-classic knowledge is the formation of rationality unity in science and philosophy. It should be recognized that ecology does not stand apart from this process, but it is in its context. So, in the ideas of P. Teiard de Charden, V.I. Vernadsky, N.N. Moiseyev and other thinkers and also in intelligent investigations of the Roman Club we perceive the aspiration to integration within the limits of ecological knowledge of scientific and philosophical kinds of investigations. For instance, N.N. Moiseyev pointed out to similar integration, supposing that philosophy transforms disembodied knowledge of human nature into "metascience of a place, purposes, possibilities of human existence as an organic part of the universe $\gg[4]$.

In a certain sense namely the sign of a post-nonclassic stage in the development of modern ecology for us is N.V. Popkova's Monograph «Philosophical Ecology » [4]. The topicality of this work is dictated by the fact that the development of different ecological subjects requires now the formation of merely the philosophical, in a sense, integral ecology [4]. In other words, besides rapid development of a variety of modern ecology branches, the intensive activity concerning our knowledge extension in the field of philosophic ecology is necessary.

In this connection in this work we set an object to show what, to out mind, is hidden behind the comprehension of post-non-classical ecology as a symbiosis of scientific and philosophic knowledge. For this, apparently, it makes sense to see what the reflection of basic features of post-non-classical scientific knowledge reveals in a current ecological discourse and what sense they are loaded with. Realizing a similar strategy we address ourselves to some fundamental philosophical principles and genesis and, through the prism of all this, to the problems of philosophy.

As is well known, the great German philosopher, E. Kant, has defined a person as «a resident of two worlds » - the outer world and the world of his/her own soul. The problem of the relation of these two worlds is ancient. A person, as a matter of fact, is interested in the relation of these two worlds, the inhabitant of which according to Kant s/he is. The way, how these existing worlds are correlated in the time of human life, is the problem which is by no means useless, and in certain situations is painful and even morbid.

Being unable to describe a mutual determination of both worlds and, in such a way, to solve a problem of their relation, philosophy increasingly began to oppose them to each other and describe them separately. Just on the basis of this contrasting the confrontation of two basic branches of a classical stage in the development of philosophy idealism and materialism was formed, and, also the adherents of this or that philosophic doctrine, so called idealists and materialists, appeared. If the former considered inner world to be primary, the latter prioritized material world. Today the problem of the spirit and matter relation, of consciousness and existence, seems to become obsolete. At the same time it is significant to comprehend the confrontation of idealists and materialists, on the one hand, in advanced science (owing to « bifurcation » of the world into ideal and material, it turned out to be possible in one way or another to comprehend the structure of each world of our interest), but on the other hand, for quite some time now it began to apply the brake to science (as the confrontation of ideal and material worlds as though tabooed the searches of logic of their mutual determination). Such is an inconsistency of comprehension: when carrying out an analytical division of the world into parts, it makes possible the comprehension of these parts, but complicates the comprehension and their interactions and their joint existence as the very integral phenomenon. And at the same time for modern philosophy and science the integral comprehension becomes utmost urgent as an indirect evidence of the development of synergy, methodology of multi-disciplinary investigations, the complexity theory and so on.

In the era of the classic development of philosophy and science it turned out that the material world is simpler in perception than the inner one. It yields easier and is better for formalization and structuring. Owing to this fact, in classical science natural history developed quicker than liberal arts. Under this influence even in the XX-th century in many sciences solely natural scientific directions proceeded with domination, while liberal arts seemed to be of the secondary importance for a long time. Such a thing occurred, for example, with geography, where for a long time namely physical geography dominated [6]. As a matter of principle, in a similar way the case was with ecology reduced to its biological branch.

The formation of the non-classical paradigm of philosophizing among other things represented a transition to the comprehension of the ways of correlation between the inner world of man and its social environment. The problem of their relation is a specific sociological projection of the problem of the relation between inner and material worlds. The rise of interest to this problem should be connected with the name of the outstanding French philosopher-positivist, O. Cont, who became a progenitor of sociology.

From the point of view of philosophy a person has a triple substance: simultaneously social, psychical and biological. In a certain sense on the basis of this idea three branches of non-classical philosophy were formed: rationalism, irrationalism and psychological direction. Each of the branches was drawn towards one of the components of the triple human substance : rationalism to the social essence (O. Cont, M. Weber, G. Simmel, Yu. Habermas et al.), irracionalism to biological essense (A. Bergson, F. Nietzsche et al.), psychological direction - to psychical (L. Word, G. Tard, V. Pareto et al.).

The non-classical philosophy did not give a possibility to describe the correlation of the ideal and material worlds, but it turned out to be humanitarian (human-centered) in its essence and gave an appreciable pulse to the development of social-humanitarian knowledge. By chance, just in the course of the nonclassical philosophy as a self-dependent science, sociology, politology, and psychology, which earlier developed solely within the limits of philosophy (as 
social philosophy, political and so on), arose. On the one hand, the positivism sought to reduce social humanitarian knowledge to natural science, but on the other hand, by means of the efforts of similar reduction it brought something new into social humanitarian knowledge, the result of which turned out to be of sociology origin. The positivism, for instance, turned the humanitarian knowledge to empiric investigations for comprehension of the world by means of accurate and methodical observations. The growth of the significance of social, humanitarian, anthropological and psychological factors and approaches to such sciences as geography, ecology, medicine was under the impact of non-classical philosophy development.

The post-non-classical science and philosophy performed the next step on this way stretched in centures. They followed not only the way of integration with each other that caused the phenomenon of general scientific knowledge, but also followed the way of the negotiation of many demarcation lines inside the field of scientific philosophical knowledge. In philosophy a process of a certain model formation of the interaction of the inner and material worlds began - the interface between spirit and matter - as it was called by one of the founders of synergetics I.R. Prigozhin. If earlier it was possible to suppose and postulate the connection of the inner and material worlds only from philosophical positions, but now the problem, concerning the investigation and modeling of this connection, arose.

It is in this context that the process of the integration of natural, technical and engineering and socialhumanitarian knowledge began. In this matter, synergetics, as it was already partly emphasized, is one of the striking examples of that in scientific knowledge border lines; in the former times its structuring affected less and less.

As a result, it is possible to suppose that one of the features of post-non-classical ecology must be, and apparently, becomes the negotiation of borders between natural and socio-humanitarian ecology. In this case, to tell the truth, a certain paradox arises. The essence of it consists in that the negotiation of borders inside of ecology must pass not through the issues weakening the ecological knowledge in different directions (though, we admit, it is possible), but through the detection and systematizing connections between those problemthematic areas which are in the center of attention of different directions of ecology. That is, as a matter of fact, there must be a modeling interface between spiritual and material aspects of the ecological reality and this, in its turn, can contribute not to elimination of borders between them, but vice versa, to their strengthening for the purpose of the reconsideration of the whole complex of ecological problems and connections inside of it.

What does all this mean?

In the first place, it means that it is necessary to consider ecological issues not only in the natural (natural - scientific) aspect, but also in the social - humanitarian one. Secondly, which is equally important, it is expedient to comprehend most completely the connection between them, their mutual determination not only within the limits of ecology to combine two ecological problems (natural and social-humanitarian). Some elementary ideas aimed at the solution of this problem we will try to formulate in this paper.

So, within the bounds of the post-non-classical ecology comprehended well enough there is an essential search and formation of such conceptual models which would not simply combine humanitarian and natural ecological issues, but determine the connection between them, and also mutual determination between the inner and material worlds in general. Solving this problem, it is evidently necessary to take into account not only ecological, but, in a sense, an extra-ecological (offecological) reality. From our point of view, within the bounds of post-non-classical ecology there is a sense to proceed from the following conceptual principles and prerequisites :

1. The ecological issues (in this case the set of problems of ecology is treated as the environment of human being, society, flora and fauna) are the result of human technical activity, engineering development, realised by a person. The engineering formation began still from time immemorial, say, with the search of tools for the world development, but in the course of time the engineering by its effect upon the world took more and more a system and systematic character, became increasingly scaled affecting it, generating and increasing ecological problems of most various kinds and sense.

2. In their developed kind of technics and technology there is a result of science, which is a certain sort of unfolding, realization, even alternative existence (other existence) of science. Engineering, in particular based on science, has become a powerful tool of the person's influence upon the world surrounding him/her with all the ensuing consequences, of both a positive and negative character. It is even possible to say that science and engineering literally pulled a person out from the natural world and opposed a person to nature. But it will be correct not in everything, as the process of man extracting from nature and the juxtaposition of man to nature was caused by culture. The perception by human society of its own unity based on the connection with culture developed hand in hand with the opposition of this community to fauna and nature in general. In this connection it makes sense to suppose that science and technology became such tools created by people which were required for the discovery of power over the forces of nature by a person. In this sense it is inportant and, in its turn, logic to consider the following point.

3. Science and, due to it, engineering (if the matter concerns engineering based on science) are the result of the human inner world, the alternative existence (otherexistence) of this world.

In a broad interpretation of the ecological reality the human inner world should be considered as its part (at the minimum - considerable, at the maximum decisive).

4. Ecological problems arising under the influence of science and engineering development cause social problems, directly and indirectly affecting the human inner world. As we can see the circle is enclosed. The 
human inner world generates science, engineering and technology, but the person her/himself becomes a hostage of his/her subject-adherence, because ecology changes result in specific social and humanitarian problems.

So, from our point of view, ecological contradictions should be analyzed taking into account rapid development of technics and technology and also technic-technological knowledge. All power of modern techno-sphere becomes the incarnation of scientific knowledge, its development and realization and, in a certain sense, an alternative existence of scientific creation, and in the end - human inner world. In this connection, ecology goes out not only in the sociotechno-natural context, but in the wider context anthropo-post-socio-techno-natural one. In any case we obtain the following chain of interconnections : " human inner world $\rightarrow$ science $\rightarrow$ technics and technology (techno-sphere, if the matter concerns general problem) $\rightarrow$ ecology problems $\gg$.

5 In a number of elements of the purpose mentioned above, it is necessary to distinguish and take into account the availability two components: natural and social humanitarian.

In particular, science, in the epistemological sense, disintegrates into natural and socio-humanitarian scientific knowledge. More than this, in the modern world technics and technology, techno-sphere, as a matter of principle «bifurcates» into «natural » technics and «social-humanitarian » one. If the first one relies upon the laws of nature, then the second - upon the regularities of the social-humanitarian plan. A striking example and indirect proof of «unnatural» technics are various social humanitarian technologies (political and social technologies, educational and marketing technologies, psycho-technics and so on) and their wide spreading in the modern world. Such sorts of technology are rather often considered as a consequence of the translation of a technical thinking style into a social-humanitarian sphere, that is, in the sphere of the existence of a person and society, in the sphere of functioning of individual and social consciousness. As one of the substantiations for such a widening approach to technics we can give the position of the well-known German philosopher and sociologist, T. Adorno. He insisted, in particular, upon the following: « a concept of spiritual culture rejecting technics arises merely from the ignorance of the society own essence. All spiritual has technical elements : only that one, who knows spirit as an observer can deceive oneself that spiritual products came out of the blue » [4].

As long as science and right after it technics bifurcate into two components (natural and social - humanitarian), then, in a similar way ecology bifurcates. In this case a two-component comprehension of ecology supposes that besides a common natural-scientific direction in ecology it is necessary that social - humanitarian ecology should exist. We are prone to connect it with such intelligent and widely interpreted approaches as ecology of human inner existence, art and culture, public consciousness, social information environment and so on. We repeat ourselves, as if a circle closes : the human inner world by means of scientific - technical and other creation transforms the environment of a person and as a consequence of this generates ecological problems and contradictions. The last ones, problems and contradictions of ecology in its wide interpretation change a person, starting all this circular (closed) process and his/her inner world.

In a similar context the necessity to address the concept of autopoiesis arises. It was developed at the beginning of the 1970-s by Chilean neuroscientists U. Maturana and F. Varela. From their point of view, all living substances are autopoetic, that is, reproducing themselves [2].

The autopoiesis concept intersects with the basic provisions of synergetics, evolution epistemology , theory of complexity and other directions of the development of modern science and philosophy.

We discussed the model of ecological reality within the bounds of which the human inner world (both a person and his/her inner world exist in ecological environment, though understood widely enough) in creation and production activity (economy) changes human environment (both natural and spiritual) that has a direct impact on a person and on the human inner world. It is evident, that this model of ecological reality intersects with the idea of autopoiesis itself. In such a way, post-non-classical ecology must be accordant to the concept of autopoiesis and to a considerable extent it must rely upon it, using its achievements.

By virtue of nearness of this concept to synergetics and other phenomena of post-non-classical knowledge emphasized above, it seems to us important to emphasize that, within the limits of post-non-classical ecology, particular attention should be paid to the following intellident investigations :

- to the comprehension of effects of self-organization and its limits in ecology;

- to the searches of management phenomena and boundaries of controllability in ecological reality;

- to the works in the field of epistomological and cognitive ecology investigating the influence of human knowledge and human inner world upon ecology and responding ecology influence upon man and human inner world.

In any case in the corresponding context it becomes evident that reflecting on similar logic we are just in the field of post-non-classical knowledge. This is evidenced by the fact that the conceptual model offered by us points out the autopoiesis of both human inner world (which changes through one's own creation of both the environment and itself), and ecological reality in its broad interpretation. Not for nothing, one of the basic conditions of the autopoiesis concept supposes coevolution of an autopoiesis system and its surroundings [2], in our case - inner world and the universe in general.

The post-non-classic side of an ecological approach considered by us, in addition, witnesses a fractal character of the offered conceptual model of ecological reality (but fractals, to the considerable extent, are addressed to synergetics and point out chaotic dynamics). 
The fractality of ecological reality in the interpretation supposed by us becomes apparent in that, on the one hand, human inner world, as well as a person himself/herself is a part of the real world, that is, of a certain ecological environment in this or that its comprehension. And, on the other hand, this environment turns out to be an object of the influence of human inner world. Fairly, human inner world seems as if it were imbedded in the real world, but subjectively the human inner world contains the real world image, that gives the possibility to influence the latter according to that map which we call the image of the real world and which, to some extent is developed by science. With something significant it reminds two mirrors installed opposite each other, where each of them is reflected in the other, and, in a certain metaphorical sense, is a part of each other. This is our case: the human inner world is a part of the material world and the material world find its reflection in the human inner world.

\section{Conclusion}

So, we are facing not a usual control situation when the subject of control (a control system, in this case, the human inner world) and the object of control (a controlled system, in this case, ecological environment) have direct and reverse connections, but something more complicated. Not without a reason T.V. Chernigovskaya, a Russian researcher, pointed out, «the brain is in the world and the world is in the brain, and to the greater extent is defined by it » [5], that is, the world and consciousness are like mirrors from the interpretation example shown above. May be, this principle should be recognized as one of the most important postulates of post-non-classical ecology.

Returning a little bit back we emphasize: we proceed from the fact that the ecological problems demand understanding just in contexts wide enough for a philosophic and anthropo-social sense. As a significant reason for such an approach we are prone to consider Kant's definition of a person as « an inhabitant of two worlds », which became classic. It is our deep conviction that the solution of ecological problems in their widest comprehension cannot be solved beyond the development of the ideas about a connection system available between micro- and macrocosm, ideal and material. Beyond logic elucidation of mutual determination of «two worlds" and beyond comprehension of the phenomenon of their mutual direction at the level of the universal determination, one cannot comprehend sufficiently the logic of complicated and contradictory evolution of the ecologic reality. As a significant reason of the approach developed by us is an idea of already mentioned triple nature of a person (psychical, social and biological). In the similar context it should be expedient to point out the triple nature of human ecology: besides biological aspects of this field of ecology, it is necessary to take into account that there is psychological ecology and social one.

To the considerable extent our approach could be considered as one of the variations of a so-called socio- techno-natural approach. It should be perceived in an aspiration to discover within the limits of the ecology of connections of social, man-caused (anthropogenic) and natural worlds. At the same time, the variation of a socio-techno-natural approach, offered by us, is an element of post-non-classic investigations of scientificphilosophic rationality and is based on the idea of changes of paradigms of scientific and philosophic thought ( « classic $\rightarrow$ non-classic $\rightarrow$ post-non-classic »).

It is possible to speake about post-non-classic ecology as a phenomenon of post-non-classic knowledge in some aspects :

- in the sense that it is aimed at the definition of interconnection between ideal and material worlds and on this basis at the negotiation of microcosm and macrocosm;

- in the sense that within the limits of it a question of harmonization in relations of microcosm and macrocosm arises;

- in the sense that the model of ecological reality and interaction of human inner world and outer environment have an autopoietic and fractal character.

What else could be urgent for post-non-classic ecology? We suppose that the development of visual ecology as a result of strengthening the language of images in the structure of ecological knowledge is urgent. A certain sort of topicality could present the seach and study of net phenomena in ecology and also the development of a kind of microecology.

So, we tried to formulate certain ideas which, in our deep conviction, must be reflected within the limits of the post-non-classic stage of the development of ecological science. Thus, the ideas offered could require definitions, but that is just the point, that they should be developed, more precise, verified. In principle, one should carry out searches of more successful conceptual approaches, including those aimed at the alignment within the limits of ecology their specific variant of interface between matter and spirit. Besides, from these ideas there must be held the transition to more concrete models, in which connection, of both an empiric, and theoretical character.

\section{References}

1. V.M. Rosin, Epistemology \& Philosophy of Science, 1 (XI), 113-115 (2007)

2. E.N. Knyazeva, Bulletin of Tomsk State Pedagogical University, 1, (2008)

3. J. Delez, F. Guattari, What is Philosophy? (Moscow, Institute of Experimental Sociology; Saint Petersburg, Aleteiya, 1998)

4. N.V. Popkova, Philosophic Ecology (Moscow, LIBROKOM, 2010)

5. T.V. Chernigovskaya, Journal Club «Intelros», 1, (2014)

6. Yu.N. Gladky, Regional Investigations, 3, (2010) 